Instituto Internacional de Investigación y Desarrollo Tecnológico Educativo INDTEC, C.A.

DOI: https://doi.org/10.29394/Scientific.issn.2542-2987.2018.3.8.9.181-196

OAI-PMH: http://www.indteca.com/ojs/index.php/Revista Scientific/oai

\title{
Sensibilización en la Manipulación de Basura en Educación Inicial
}

Autora: Santa Yoraima Monzón Bravo Universidad Pedagógica Experimental Libertador, UPEL santamonzon1984@gmail.com

Barinas, Venezuela

\section{Resumen}

El estudio se direccionó mediante un objetivo de formular un plan de sensibilización en la manipulación de la basura en el Centro de Educación Inicial Bolivariano "Cinqueña III" del municipio Barinas estado Barinas. El enfoque investigativo empleado fue el cuantitativo, a través de un proyecto factible con un diseño de campo. El universo poblacional lo constituyen doce (12) profesionales en la docencia, veinte (20) padres y representantes del mencionado centro educativo, los cuales fueron seleccionados porque pertenecen al mismo contexto de estudio, la muestra fue el cien por ciento (100\%). Los datos se obtuvieron por la aplicación de dos cuestionarios con de veinte (20) preguntas cada uno con tres alternativas siempre, algunas veces y nunca, validándose mediante el criterio de expertos y para la confiabilidad se utilizó una prueba piloto a través del coeficiente de Alfa de Cronbach con un resultado de 0.846 . Las derivaciones se reflejaron en cuadros y gráficos para obtener una visión conclusiva en la cual los actores sociales tomaron conciencia sobre la manipulación de la basura por medio de actividades y estrategias para cambiar actitudes y hábitos de consumo en la población objetivo.

Palabras clave: gestión ambiental; comunicación en grupo; tratamiento de desechos. 


\title{
Sensitization in the Handling of Garbage in Initial Education
}

\begin{abstract}
The study was addressed through an objective to formulate a plan of awareness in the handling of garbage in the Bolivarian Initial Education Center "Cinqueña III" of the Barinas State Barinas. The investigative approach used was quantitative, through a feasible project with a field design. The population universe consists of twelve (12) professionals in teaching, twenty (20) parents and representatives of the aforementioned educational center, which were selected because they belong to the same study context, the sample was one hundred percent $(100 \%)$. The data was obtained by the application of two questionnaires with twenty (20) questions each with three alternatives always, sometimes and never, validated by expert criteria and for reliability a pilot test was used through the Alpha coefficient of Cronbach with a result of 0.846 . The referrals were reflected in charts and graphs to obtain a conclusive vision in which the social actors became aware of the manipulation of waste through activities and strategies to change attitudes and consumption habits in the target population.
\end{abstract}

Keywords: environmental management; communication in group; waste treatment. 


\section{Introducción}

Las demandas enfrentadas por la educación, durante el devenir del siglo XXI, se ha caracterizado por ser responsables ante el ambiente, tomando en cuenta a la conservación como uno de sus elementos, mediante el compromiso de cuidar y preservar los recursos, además de adquirir una conciencia ambientalista en la cual participen responsablemente los ciudadanos. De allí, estos aspectos han sido considerados por las leyes vigentes como un derecho y un deber combinado con la interacción de los actores educativos para la transformación del medio.

Desde esta perspectiva, se ubica la posibilidad de introducir modificaciones en el medio valores ambientales que brinden oportunidades tanto a docentes como niños para hacer un uso adecuado de éste asumiendo actitudes conservacionistas que involucre la manipulación de la basura a través de su reutilización, para transformar desechos en materiales utilitarios que pueden ser empleados durante el desarrollo de las actividades en los diferentes espacios de aprendizaje. Este planteamiento, surgió la interrogante: ¿Cuáles son los requerimientos de un plan de sensibilización del personal docente y comunidad sobre la manipulación de basura en el Centro de Educación Inicial Bolivariano "Cinqueña III" del municipio Barinas estado Barinas? Permitiendo la realización de una investigación con el objetivo de formular un plan de sensibilización en la manipulación de basura en el Centro de Educación Inicial Bolivariano "Cinqueña III" del municipio Barinas estado Barinas

\section{El Problema}

La sociedad contemporánea demanda de acciones para superar problemas ecológicos conjuntamente con la crisis ambiental surgida por la creciente industrialización en los diferentes ámbitos de la vida, las cuales ocasionan un impacto negativo en la calidad del ambiente, reflejada sobre la 
base de una elevada contaminación. Por tanto, corresponde a las instituciones educativas, asumir una educación ambiental como un proceso de participación con la actuación permanente de los actores socioeducativos que intervienen en el sistema educativo.

En efecto, se concuerda con los planteamientos realizados por Boada (citado en Briceño y Romero, 2007a): quien la considera como "un proceso permanente en el que los individuos y la comunidad se conciencian de sus destrezas, experiencia, también la determinación que les permitirá actuar individual o colectivamente para resolver los problemas ambientales presentes y futuros" (pág. 493); involucrando, por consiguiente, a docentes, niños, padres, representantes, miembros de la comunidad, entre otros. De allí, se hace evidente la necesidad de introducir cambios en el ejercicio del docente que conduzcan hacia una práctica sustentada en la gestión del ambiente.

De esta manera, la gestión ambiental está acompañada de acciones para la conservación de los recursos, mediante actividades direccionadas hacia un manejo integral de los residuos sólidos e introduciendo la reutilización de productos en la elaboración de materiales que pueden ser empleados como recursos para el aprendizaje en las instituciones educativas. Sobre el particular Bureau Veritas Formación, citado en Sáez, Leal y Monasterio (2014a): acota "la reutilización se define como la acción de volver a utilizar los bienes o productos desechados, con el mismo fin para el que fueron diseñados, es decir, dar a los materiales más de una vida útil" (pág. 122); implicando, así, acciones que preservan el ambiente y favorecen las condiciones de los miembros en la institución educativa.

Por tanto, la educación es determinante en la formación de los individuos, razón por la cual las acciones conservacionistas deben introducirse durante la niñez, es decir, en la formación preescolar, porque el infante progresivamente toma conciencia del entorno en que se desenvuelve; además, va adquiriendo dominio de las habilidades contribuyentes en la 
configuración de su madurez tanto intelectual como afectiva. Asimismo, el Centro de Educación Inicial constituye el espacio para la internalización de un conocimiento que lo conduzca a tomar decisiones a los problemas que enfrenta en una sociedad dinámica. Por ello, corresponde al docente crear en los preescolares una conciencia ecológica para darle un uso adecuado a los recursos naturales de manera que los conserve y preserve de posibles daños que lo desequilibren.

Tomando como referencia lo expuesto, se plantea la necesidad de una investigación que sea expresión de las preguntas: ¿Cuáles son los requerimientos en un plan de sensibilización del personal docente y comunidad sobre la manipulación de basura? ¿De qué se puede determinar la factibilidad en la propuesta orientada a la concienciación en los actores educativos y comunidad sobre el manejo de residuos y desechos sólidos?

Igualmente, se consideró ¿Cuál será el diseño de la propuesta de un plan antes referido dirigida a los miembros del personal docente y comunidad? ¿Cómo aplicar las acciones planificadas? ¿Cuál sería el proceso para la valoración de los beneficios en la realización de las actividades?

\subsection{Intenciones Investigativas}

\subsubsection{Intención Rectora}

Formular un plan de sensibilización en la manipulación de basura en el Centro de Educación Inicial Bolivariano "Cinqueña III" del municipio Barinas estado Barinas.

\subsubsection{Intenciones Concretas}

Diagnosticar los requisitos de un plan de sensibilización del personal docente sobre la manipulación de basura.

Determinar la factibilidad en la propuesta de la intención de sensibilización para el talento humano sobre la utilización de basura. 
Diseñar plan de sensibilización del personal docente sobre el empleo de la basura en la institución educativa estudiada.

Cabe señalar, que el presente proyecto de trabajo de grado se encuadra con la línea de investigación: Educación, Desarrollo y Gestión Comunitaria, que sirve de cimiento a los subprogramas de Maestrías en el Núcleo Académico Barinas de la Universidad Pedagógica Experimental Libertador.

\section{Perspectiva Empírica}

\subsection{Estudios Previos}

En correspondencia a los estudios previos considerados como antecedentes investigativos relacionados con el tema en estudio, Palella y Martins (2012a): señala "los antecedentes de la investigación, entendida como diferentes trabajos realizados por otros estudiosos sobre el mismo problema" (pág. 63); para lo cual se realizó una revisión de tesis de postgrado, investigaciones institucionales, revistas especializadas, memorias de congresos, entre otras.

Así, Sáez, Leal y Monasterio (2014b): en un trabajo titulado: "Residuos sólidos en las instituciones educativas", con el objetivo de conocer el comportamiento de la cantidad de residuos sólidos en Kilogramos por día ( Kg/día) generados en las instituciones educativas del municipio Maracaibo del estado Zulia, respaldada en fundamentos positivistas, como del constructivismo ecológico, además de nociones y compendios reglamentarios del reaprovechamiento en educación.

De igual manera, Briceño y Romero (2007b): realizó un trabajo denominado: "Formación de valores en educación ambiental para la conservación del ecosistema", con el objetivo de analizar la formación de valores en educación ambiental para la conservación del ecosistema en la asignatura Educación Ambiental de la Licenciatura de Educación Integral de la Universidad Nacional Experimental Rafael María Baralt; metodológicamente 
se ubicó como una investigación de campo y del tipo descriptiva.

Consecuentemente al análisis se estableció que los motivos porque no se animan trabajos que impulsen valores ambientales en la escuela, son por la escasa intervención en las decisiones emprendidas y el conocimiento para el resguardo y preservación del medio.

\subsection{Bases Teóricas}

Las bases teóricas se direccionaron en función de un plan de sensibilización, entendido como un proceso estructurado para el diseño y organización de acciones que vayan a concienciar a los miembros de la institución, por consiguiente, es un instrumento de planeación, que refleja las estrategias y directrices a seguir por la institución. Al respecto, Benjumea y Carrión (2011): lo define como "un conjunto integrado de objetivos, metas y actividades en el marco de las políticas medioambientales" (pág. 4); lo cual implica una planificación que se diseña para conseguir transformar la realidad.

Así, se orienta hacia el mejoramiento de las condiciones educativas mediante la coordinación de las actividades para la identificación de situaciones que requieren de un cambio en las actitudes y comportamientos de los involucrados. Además, suministra un escenario para la introducción de aspectos relacionados con la vigilancia, valoración, búsqueda y monitoreo de las acciones; pudiéndose ejecutar actividades que beneficien la calidad del medio tanto en las escuelas como den la comunidad en la cual se inserta mediante la reutilización de los desechos sólidos.

En este orden de ideas, se tomó como referencia respecto a los desechos sólidos la clasificación realizada por Bustos (2009): quien los sitúa como "orgánico e inorgánico" (pág. 12); estructurándose la reutilización de residuos orgánicos, hacia la descomposición controlada de frutas, verduras, pasto, hojas, entre otros; es decir, que alcanzar su desintegración o putrefacción mediante la acción de microorganismos, oxígeno y factores 
climáticos; pudiéndose convertir en abono orgánico para las plantas. En tanto, la reutilización de desechos inorgánicos se descompone de manera natural, razón por la cual pueden ser reusados o transformados a través de métodos artificiales o mecánicos.

\section{Marco metodológico}

En relación con los procedimientos que conforman la metodología implementada en el proceso investigativo, se asume lo esbozado por la Universidad Pedagógica Experimental Libertador, UPEL (2016a): afirma "se describen los métodos, técnicas y procedimientos aplicados de modo que el lector pueda tener una visión clara de lo que se hizo, por qué y cómo se hizo" (pág. 34); implicando, de esta manera los procesos realizados para la adecuación del problema a la realidad estudiada.

\subsection{Diseño de la investigación}

Las ideas precedentes, conducen a destacar el proceso investigativo se desarrolló bajo el enfoque positivista, encuadrado en un proyecto factible, respaldada en una investigación de campo de tipo descriptiva, no experimental y transeccional. Respecto a la modalidad, al parafrasear al Manual de Trabajos de Grado de Especialización y Maestría y Tesis Doctorales de la Universidad Pedagógica Experimental Libertador, UPEL (2016b): radica en la exploración, producción y perfeccionamiento de una proposición de una representación posible para corregir dificultades, exigencias o insuficiencias mediante la enunciación de habilidades, métodos, técnicas o conocimientos.

Es importante subrayar, esta modalidad debe cumplirse en las fases que Palella y Martins (2012b): plantean como de diagnosis, establecimiento de elementos teóricos como procesos metodológicos para la ejecución de las actividades; además, de la viabilidad, puesta en práctica de las proposiciones.

Lo reflejado por el autor, se ha de iniciar con un diagnóstico de la 
situación real objeto de estudio, seguido de una factibilidad y por último la propuesta. En la Fase I de investigación diagnóstica, mediante la exploración, examen o detección de la problemática estudiada por medio de la aplicación de procesos para obtener la información, para la verificación del problema, que en el estudio se empleará preguntas a los educadores como a progenitores.

Por otra parte, la Fase II corresponde a la elaboración de la propuesta como alternativa que pretende solucionar la problemática existente en el Centro de Educación Inicial en estudio. Mientras, la Fase III, se direccionan hacia la evaluación de la factibilidad; es decir, se determina la viabilidad como disponibilidad de recursos financieros y humanos para ejecución de la propuesta en mención.

\subsection{Población y Muestra}

En la investigación, se asumió los planteamientos de Balestrini (2006): respecto a la población, quien señala constituye el "conjunto de elementos de los cuales se pretendemos indagar y conocer sus características, o una de ellas, y para el cual serán válidas las conclusiones obtenidas en la investigación" (pág. 137); estando conformada por doce (12) docentes, así como veinte (20) padres y representantes del preescolar bajo objeto de análisis, seleccionados porque laboran en la referida institución, en tanto, a los representantes por su participación en las actividades planificadas y los compromisos como responsabilidades asumidas.

\subsection{Validación}

Para el logro de la eficacia de las preguntas, Palella y Martins (2010c): señala: "se define como la ausencia de sesgos. Representa la relación entre lo que se mide y aquello que realmente se quiere medir" (pág. 172); en tal

razón, fundamentado en esta base teórica, el cuestionario será validado a través del criterio de tres especialistas para verificar la relación existente entre 
todos los elementos referentes a la operacionalización de las variables, además realizarán las observaciones los expertos, con la finalidad de reajustar los ítems.

\subsection{Análisis de datos}

El uso de técnica de análisis de datos, las cuales son definidas por Sabino (2014): como "procedimientos que son sometidos los datos para verificar los resultados de la investigación" (pág. 185); que, en este caso, se realizará por medio de la estadística descriptiva, tomando como base la elaboración de cuadros de distribución de frecuencias. Respecto al instrumento aplicado a los docentes, los resultados obtenidos se muestran en el Cuadro 1.

Cuadro 1. Distribución Frecuencia y Porcentaje en relación con la Variable: Plan de sensibilización para el manejo de residuos sólidos en su Dimensión: Clasificación para el Indicador: Papel y Cartón. En el Centro de Educación inicial se clasifican los residuos sólidos provenientes del papel y cartón.

\begin{tabular}{|l|c|c|}
\hline \multicolumn{1}{|c|}{ Opción } & Frecuencia & Porcentaje \\
\hline Siempre & 0 & $0 \%$ \\
\hline Algunas veces & 0 & $0 \%$ \\
\hline Nunca & 12 & $100 \%$ \\
\hline \multicolumn{1}{r|}{ Total } & $\mathbf{1 2}$ & $\mathbf{1 0 0 \%}$ \\
\hline
\end{tabular}

Fuente: Instrumento aplicado por la Autora (2017).

Respecto al cuadro 1, el (100\%) de los docentes opinaron que "Nunca" en el Centro de Educación Inicial se clasifican los residuos sólidos provenientes del papel y cartón, los cuales son almacenados temporalmente para su clasificación y depositados en recipientes que a juicio de González, Muñoz y Parra (2015a): permiten su separación como "orgánicos, reciclable y no reciclables o desechos en el sitio de origen" (pág. 39); convirtiéndose, en 
un mecanismo que puede agregar valor a los desperdicios provenientes del papel y cartón. Igualmente, en el Cuadro 2 se muestra.

Cuadro 2. Distribución Frecuencia y Porcentaje con relación a la Variable: Plan de sensibilización para el manejo de residuos sólidos en su Dimensión: Ciclo del Reciclaje para el Indicador: Reducir. En la institución se realizan acciones para reducir la existencia de desechos sólidos.

\begin{tabular}{|c|c|c|}
\hline Opción & Frecuencia & Porcentaje \\
\hline Siempre & 0 & $0 \%$ \\
\hline Algunas veces & 0 & $0 \%$ \\
\hline Nunca & 12 & $100 \%$ \\
\hline Total & 12 & $100 \%$ \\
\hline
\end{tabular}

Fuente: Instrumento aplicado por la Autora (2017).

Observándose en estos resultados que el (100\%) de los docentes opinaron que "Nunca", en la institución se realizan acciones para reducir la existencia de desechos sólidos. Esto trae como consecuencia problemas contaminantes dentro y fuera de la institución, por lo que se debería tomar cartas en el asunto al crear conciencia ambientalista, donde docente y comunidad ayuden a solucionarlos, de allí que la reducción de desechos sólidos según González, Muñoz y Parra (2015b): señalan que reducir, se realiza con "la finalidad es disminuir el gasto de materias primas, agua y bienes de consumo, así como el aporte de $\mathrm{CO} 2$ a la atmosfera y el consumo de energía (tanto la destinada a la creación de un producto como a su transporte y distribución)" (pág. 36).

\section{Visión Conclusiva}

Desde el punto de vista de la investigadora, se considera de vital importancia el tratamiento de los valores ambientales en las instituciones educativas, en especial en los Centros de Educación Inicial porque introduce 
al niño en el espacio de la preservación del medio; además, crea en el infante una responsabilidad hacia la preservación de los recursos, haciendo uso de acciones como la manipulación de basura; trascendiendo así, la cultura imperante en las comunidades caracterizada por la existencia de residuos considerados como basura que pueden ser transformados y aprovechados como recursos para el aprendizaje.

Es importante señalar, la manipulación de basura forma parte de los contenidos de Educación Ambiental, que a juicio de González (2017): debe ser asumido como "un proceso educativo que enfatiza la sensibilización de los problemas ecológicos y socioculturales que permitan la promoción de acciones con carácter preventivo y remedial" (pág. 108), lo cual implica, el reconocimiento de los valores inherentes al área, orientados hacia la preservación y conservación del entorno.

De este modo, se puedo diagnosticar los requisitos de un plan de sensibilización del personal docente sobre la manipulación de basura, sustentándose en función de conocimientos, habilidades y actitudes que favorezcan una cultura hacia el manejo de desperdicios hasta lograr su reutilización; partiendo de un comportamiento ambiental responsable. En este sentido, se derivó una práctica educativa que consideró las variables o entornos y las interacciones que influyen para que el desarrollo se produzca en forma integral y armónica: la familia, la escuela conjuntamente con la comunidad.

Considerando los planteamientos anteriores, se determinó que el plan de sensibilización formulado se considera viable desde el punto de vista técnico, por la presencia de personal altamente calificado y capacitado dentro del municipio Barinas como en el Estado Barinas, quien desea colaborar en el desarrollo de iniciativas que mejoren sustancialmente las condiciones ambientales en las instituciones educativas; además, financieramente es factible porque se estableció un programa de financiamiento, que incluyó la 
cogestión de recursos con los colectivos institucionales que hacen vida en el plantel y organismos públicos como privados que existen en la comunidad.

Del mismo modo, el plan responde a las directrices estratégicas del Ministerio del Poder Popular para la Educación, dirigidas a lograr el desarrollo integral de la educación nacional, de manera que se superen los desequilibrios ambientales, sociales y culturales. Asimismo, tiene un carácter eminentemente social como educativo, porque se orienta hacia una educación integral, de calidad, que apoye la inserción y permanencia del educando en la institución escolar.

Por tanto, se procedió a diseñar plan de sensibilización del personal docente sobre el empleo de la basura en la institución educativa estudiada, en las siguientes fases:

Fase A: dirigida hacia un proceso de formación teórico-práctico que incluyó integración como participación de los actores educativos en talleres, conversatorios y difusión de los conocimientos adquiridos.

Fase B: encaminada hacia la operatividad de la propuesta acompañada de una planificación e implementación de las actividades.

Fase C: denominada control y evaluación con el propósito de valorar las actividades realizadas y por ende el plan de sensibilización.

\section{Referencias}

Balestrini, M. (2006). Cómo se Elabora un Proyecto de Investigación. Séptima. Edición. Caracas, Venezuela: Editorial BL. Consultores Asociados. Servicio Editorial.

Benjumea, A., \& Carrión T. (2011). Plan de Sensibilización Ambiental de

Santa Coloma de Gramenet. España: Congreso Nacional de Medio Ambiente. Cumbre del Desarrollo Sustentable.

Briceño, H., \& Romero R. (2007a,b). Formación de valores en educación 
ambiental para la conservación del ecosistema. Universidad Rafael Belloso Chacín. TELOS. Revista de Estudios Interdisciplinarios en Ciencias Sociales 9(3), 491-508. Recuperado de: http://publicaciones.urbe.edu/index.php/telos/article/viewArticle/3282/4 174

Bustos, C. (2009). La problemática de los Desechos Sólidos. Economía, XXXIV, 27, (enero-junio), págs. 121-144. Recuperado de: http://iies.faces.ula.ve/Revista/Articulos/Revista 27/Pdf/Rev27Bustos.p $\underline{\mathrm{df}}$

González, J. (2017). Programa para la Integración Didáctica de los Contenidos de Educación Ambiental. Revista Scientific, 2(5), 105124. Recuperado de: https://doi.org/10.29394/scientific.issn.25422987.2017.2.5.6.105-124

González, E., Muñoz, A. \& Parra, L. (2015a,b). Estrategias Lúdico Pedagógicas para sensibilizar en la gestión integral de los residuos sólidos en la comunidad educativa de la sede Ignacio Torres Giraldo del municipio Palmira, Departamento Valle del Cauca. Especialización en Educación Ambiental. Santiago de Cali: Fundación Universitaria Los Libertadores, págs. 112. Recuperado de: http://repository.libertadores.edu.co/bitstream/handle/11371/387/Gonz alezOsorioElizabeth.pdf?sequence $=2$

Palella, S., \& Martins F. (2012a,b,c). Metodología de la investigación cuantitativa. 3ra Edición. ISBN: 980-273-445-4, págs. 279. Caracas, Venezuela: Fondo Editorial de la Universidad Pedagógica Experimental Libertador, FEDUPEL. Recuperado de:

http://es.calameo.com/read/000628576f51732890350

Sabino, C. (2014). El Proceso de Investigación. 10a Edición revisada y corregida, 1aㅗ reimpresión. ISBN 978-9929677074. Guatemala: Editorial Episteme, págs. 240. Recuperado de: 
https://books.google.co.ve/books?id=jwejBAAAQBAJ\&printsec=frontco ver\&hl=es\&source $=$ gbs ge summary $r \& c a d=0 \# v=$ onepage \& $q \& f=f a l s e$

Sáez, A., Leal N., \& Monasterio S. (2014a,b). Residuos sólidos en instituciones educativas. REVECITEC: Revista Venezolana de Ciencia y Tecnología. 5(1), 1-20. Recuperado de: http://publicaciones.urbe.edu/index.php/revecitec/article/viewArticle/32 $\underline{12}$

Universidad Pedagógica Experimental Libertador (2016a,b). Manual de Trabajos de Grado de Especialización y Maestría y Tesis Doctorales. 5a Edición. IBSN 980-273-441-1. Caracas, Venezuela: Fondo Editorial de la Universidad Pedagógica Experimental Libertador, FEDUPEL. Recuperado de:

https://www.slideshare.net/mirnalitaguirrez/manual-upel-2016-1pdf 


\section{Santa Yoraima Monzón Bravo}

e-mail: santamonzon@gmail.com

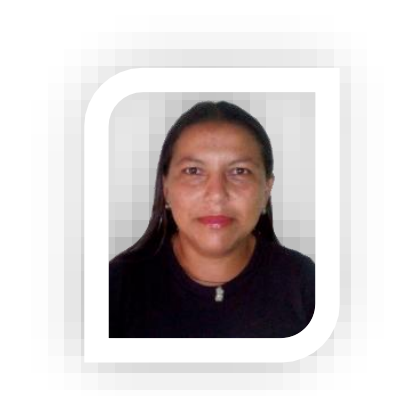

Nacida en Palmarito, estado Apure, Venezuela. Licenciada en Educación Integral (UNELLEZ, Barinas), Especialista en Gerencia Educacional, Universidad Pedagógica Experimental Libertador, UPEL-Guanare, Docente Titular en El C.E.I.B. "Cinqueña III", Participante en el curso de cuarto nivel de Formulación y Evaluación (2017). 